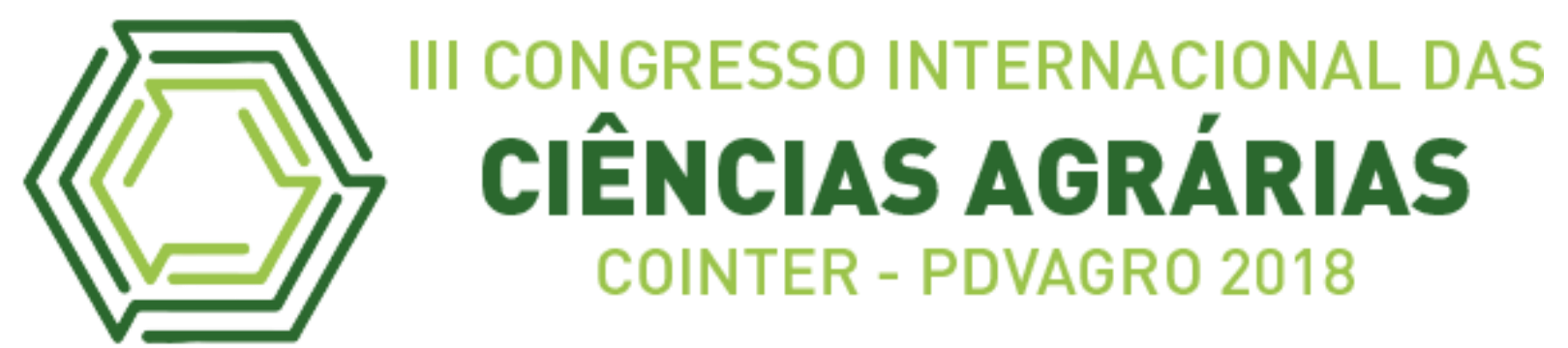

\title{
AVALIAÇÃO DA QUALIDADE DO SOLO NO SETOR DE AGRICULTURA DA UNIVERSIDADE FEDERAL DA PARAÍBA EM BANANEIRAS-PB
}

\section{SOIL QUALITY ASSESSMENT IN THE AGRICULTURE SECTOR OF THE FEDERAL UNIVERSITY OF PARAÍBA IN BANANEIRAS-PB}

\author{
Apresentação: Comunicação Oral
}

David Marx Antunes de Melo ${ }^{1}$; Ivan Sérgio da Silva Oliveira ${ }^{2}$; Stéfano Sendko ${ }^{3}$; Thiago do Nascimento Coaracy ${ }^{4}$; Alexandre Eduardo de Araújo ${ }^{5}$

\section{DOI: https://doi.org/10.31692/2526-7701.IIICOINTERPDVAGRO.2018.00046}

\section{Resumo}

A agricultura, de diferentes formas e intensidades, causa perturbações ao ecossistema natural. Métodos de avaliação da sustentabilidade de agroecossistemas são de extrema importância para o diagnóstico dessas áreas, pois de modo geral, possibilitam visões amplas e sistêmicas acerca dos impactos causados pelas práticas agrícolas utilizadas. Dessa forma, este trabalho teve como objetivo avaliar, a sustentabilidade de agroecossistemas localizados no interior da Universidade Federal da Paraíba (UFPB), campus III, Bananeiras-PB. A metodologia de avaliação foi: Método Rápido e de Fácil Acesso, que avalia a qualidade dos solos, complementarmente foram realizados testes laboratoriais. Os subsistemas avaliados foram: Manga, Mandala e a Mata Nativa que serviu como referência. O subsistema Mandala apresentou ótimos resultados sob ótica da metodologia adotada, foram também promissores os resultados laboratoriais, já o subsistema Manga apresentou baixa biodiversidade funcional, consequentemente baixos índices de qualidade dos solos. A metodologia se mostrou uma ferramenta eficaz facilitando a percepção das condições atuais de cada subsistema.

Palavras-Chave: qualidade do solo, etnopedologia, indicadores de sustentabilidade

\footnotetext{
${ }^{1}$ Mestrando em Agroecologia - UFPB - CCHSA, Campus III, davidatunes@gmail.com

${ }^{2}$ Mestrando em Agroecologia - UFPB - CCHSA, Campus III,ivantecagro@gmail.com

${ }^{3}$ Mestrando em Agroecologia - UFPB - CCHSA, Campus III,stefano.sendtko@yahoo.com

${ }^{4}$ Mestrando em Agroecologia - UFPB - CCHSA, Campus III, thiago.coaracy@gmail.com

${ }^{5}$ Doutor em Eng. Agrícola, UFPB - CCHSA, Campus III, alexandreeduardodearaujo@hotmail.com
} 


\begin{abstract}
Agriculture, of different forms and intensities, causes disruption to the natural ecosystem. Methods of evaluating the sustainability of agroecosystems are extremely important for the diagnosis of these areas, since in general, they allow wide and systemic visions about the impacts caused by the agricultural practices used. Thus, the objective of this work was to evaluate the sustainability of agroecosystems located inside the Federal University of Paraíba (UFPB), campus III, Bananeiras-PB. The evaluation methodology was: Quick and Easy Access Method, which evaluates the soil quality, complemented by laboratory tests. The evaluated subsystems were: Manga, Mandala and Native Forest that served as reference. The Mandala subsystem presented excellent results from the adopted methodology. Laboratory results were also promising, since the Manga subsystem presented low functional biodiversity and, consequently, low levels of soil quality. The methodology proved to be an effective tool facilitating the perception of the current conditions of each subsystem.
\end{abstract}

Key words: soil quality, ethnopedology, sustainability indicators

\title{
Introdução
}

O solo é um ambiente vivo com grande biodiversidade. Essa atividade biológica ajuda a determinar sua estrutura e a fertilidade sendo essencial para o desempenho de suas funções, incluindo a produção de alimentos.

A qualidade do solo é entendida como a capacidade de sustentar a produtividade biológica do ecossistema, mantendo o equilíbrio ambiental e promovendo a saúde de plantas e/ou animais e do próprio ser humano (Doran et al. 1996; Sposito e Zabel 2003). No entanto, avaliar a qualidade do solo requer o monitoramento de alguns parâmetros que variam com as mudanças no manejo ou fatores externos.

Segundo Cândido (2010), existe, atualmente, a necessidade da realização de estudos e pesquisas que levem em conta os aspectos da sustentabilidade nas atividades agrícolas, pois a agricultura é base fundamental da sociedade. Através do uso de indicadores de qualidade do solo, é possível avaliar a sustentabilidade dos agroecossistemas e de seus, pois possibilitam monitorar questões ambientais e produtivas, e quando necessário sociais e econômicas.

O objetivo do trabalho foi avaliar a qualidade dos solos de dois subsistemas produtivos e mata nativa estabelecidos na segunda Chã, do Centro de Ciências Humanas, 
Sociais (CCHSA) e Agrárias da Universidade Federal da Paraíba (UFPB), Campus III, Bananeiras, através do uso de metodologia sistêmica.

\section{Fundamentação Teórica}

O conceito de agroecossistema, compreendido como um ecossistema onde o fluxo de energia e nutrientes é, através da prática agrícola, direcionado para a produção de alimentos, fibras e demais produtos, remete a uma condição estrutural e sistêmica de análise que permite abordar a produção agropecuária de forma holística, incluindo os conjuntos complexos de insumos e produção, bem como identificar as interconexões existentes entre as partes componentes (SARANDÓN, 2014).

Os indicadores de qualidade são propriedades mensuráveis (quantitativas ou qualitativas) do solo ou da planta acerca de um processo ou atividade e que permitem caracterizar, avaliar e acompanhar as alterações ocorridas num dado ecossistema (KARLEN et al., 1997). O uso de indicadores compõe uma ferramenta metodológica capaz de mobilizar a população rural para a resolução de seus problemas (CASADO \& MIELGO, 2007).

Entretanto, devido à heterogeneidade e dinâmica do compartimento solo, a sua qualidade não pode ser mensurada diretamente, podendo ser estimada a partir de indicadores arbitrados pelo humano. As mudanças no "status" da qualidade do solo têm sido avaliadas por intermédio de sistemas qualitativos e quantitativos em que se utilizam indicadores apropriados, efetuando-se a comparação com valores desejáveis em diferentes intervalos de tempo, para um fim específico em ecossistemas diversos (KARLEN e STOTT, 1994; CHAER, 2001; MELO FILHO et al., 2007).

\section{Metodologia}

\section{Localização}

O trabalho foi realizado na primeira e segunda chã do setor de agricultura em duas áreas,subsistema Mandala e o subsistema Manga do Centro de Ciências Humanas, Sociais e Agrárias, da Universidade Federal da Paraíba, Campus III, Bananeiras - PB (Figura 1). 


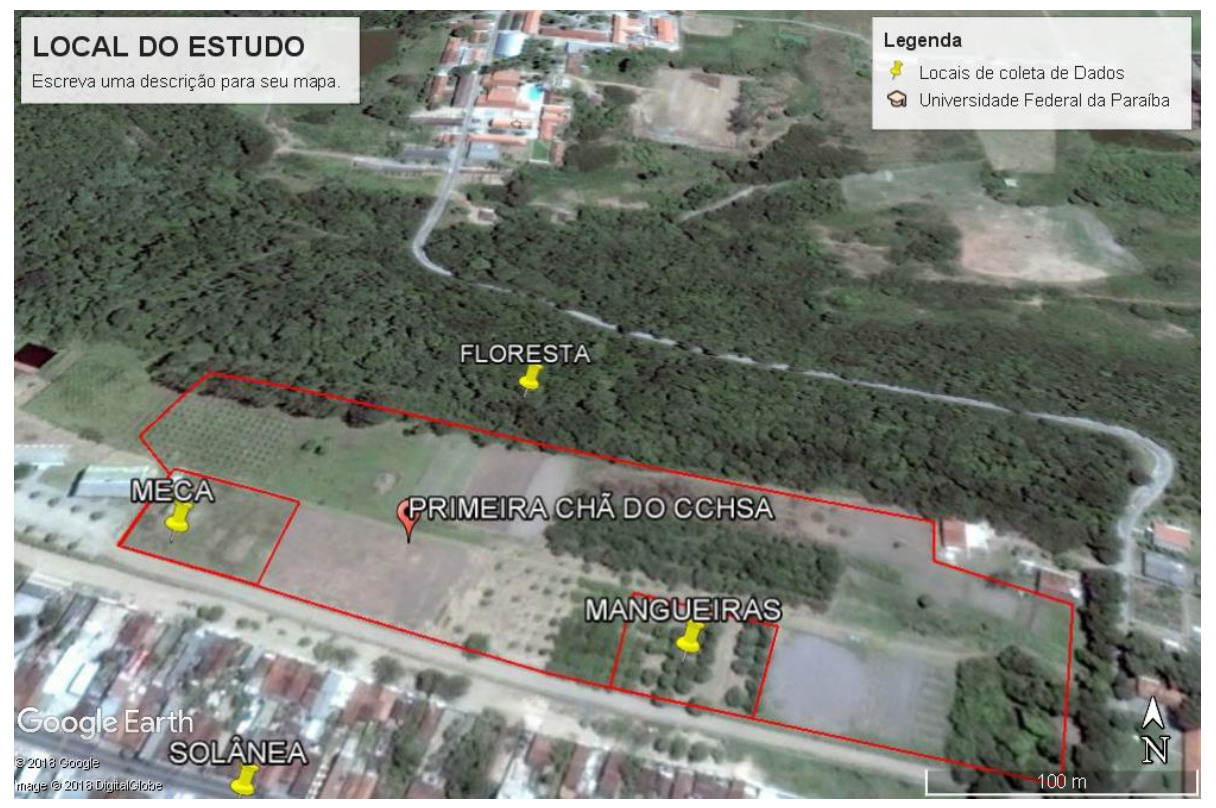

Figura 1. Local do estudo no CCHSA/UFPB. Fonte: Google Earth, 2018

\section{Questionário qualitativo}

O questionário utilizado na pesquisa foi adaptado de Nicholls et al., (2004), desenvolvido para avaliar, juntamente com os agricultores, formas mais acessíveis à qualidade do solo em diferentes sistemas. Nesse método avalia-se cada indicador da qualidade dos solos separadamente e são atribuídos valores entre 1 e 10, de acordo com os atributos observados no solo ou na cultura (1 sendo o valor menos desejável, 5 um valor moderado ou limiar e 10 o valor mais preferido). Valores entre 1 a 5 e 5 a 10 também podem ser atribuídos em conformidade (NICHOLLS et al., 2004).

Foram usados 6 variáveis, quais sejam: estrutura, compactação, erosão, matéria orgânica, cobertura do solo e atividade microbiológica, ambos foram aplicados nos dois subsistemas e na Mata nativa. Foi realizada uma análise descritiva de média e desvio padrão sendo um delineamento inteiramente casualizado (DIC) com 3 tratamentos, 6 variáveis e 3 repetições.

\section{Análise química das amostras dos agroecossistemas}

A análise química para a caracterização das amostras ocorreu no laboratório de solos do Centro de Ciências Humanas, Sociais e Agrárias (CCHSA) localizado na Universidade Federal da Paraíba (UFPB). Foi realizada uma coleta amostral composta de 0-20 cm de profundidade de cada subsistema e a Mata avaliada. As análises no laboratório, foram realizadas de acordo com o manual metodológico de análises de solo (EMBRAPA, 1997).

\section{Resultados e Discussão}


Abaixo segue os resultados da Mata e dos subsistemas, do questionário qualitativo adaptado de Nicholls et al. (2004) (figura 1) e análise química e fertilidade (tabela 1).

\section{Questionário qualitativo}

A tabela 1 mostra as médias obtidas por 3 avaliadores para cada parâmetro avaliado no experimento, onde observou-se pouca variação com relação as notas atribuídas, indicando essa metodologia como de fácil aplicação para a Agricultura Familiar.

Tabela 1. Valores médios obtidos para os atributos do solo em subsistemas produtivos e ecossistema natural localizados no CCHSA/UFPB

\begin{tabular}{|c|c|c|c|}
\hline \multicolumn{4}{|c|}{ Valores Médios } \\
\hline Atributos & Mata Nativa & Mandala & Mangueira \\
\hline Estrutura & $9,77 \pm 0,25$ & $7,20 \pm 0,17$ & $5,30 \pm 0,17$ \\
\hline Compactação & $9,83 \pm 0,29$ & $7,17 \pm 0,11$ & $5,16 \pm 0,06$ \\
\hline Matéria Orgânica & $9,77 \pm 0,06$ & $8,60 \pm 0,10$ & $6,37 \pm 0,21$ \\
\hline Cobertura do Solo & $9,90 \pm 0,10$ & $7,63 \pm 0,15$ & $6,43 \pm 0,15$ \\
\hline Pres. de Invertebrados & $9,87 \pm 0,11$ & $8,53 \pm 0,21$ & $6,30 \pm 0,10$ \\
\hline Ativ. Microbiológica & $9,90 \pm 0,10$ & $8,20 \pm 0,20$ & $5,77 \pm 0,15$ \\
\hline
\end{tabular}

Nos indicadores de Estrutura, Compactação e Cobertura do solo, a Mandala obteve o valor, sete (7), resultados que refletem as práticas utilizadas na área, tais como consórcio, rotação de culturas, uso de implementos manuais, incremento de composto e cobertura morta. A estrutura é boa indicadora da qualidade do solo devido a sua sensibilidade às práticas de manejo adotadas.

De modo geral, o solo sob manejo conservacionista com cobertura vegetal, apresenta características físicas como estrutura, permeabilidade, densidade e porosidade do solo adequadas ao desenvolvimento normal das plantas (Andreola et al., 2000). O que também afirma Primavesi (2016), para determinar a estrutura agrícola do solo, pega-se um torrão. Se este, por pressão leve, se desmanchar em grumos, o solo é ótimo...quanto mais irregular os torrões, melhor a condição do solo. A matéria orgânica, em primeiro lugar, é alimento para a vida aeróbia do solo que produz os agregados.

$\mathrm{Na}$ figura 2, é apresentado em esquema de teia, os subsistemas avaliados Manga e Mandala, em referência a Mata nativa. 


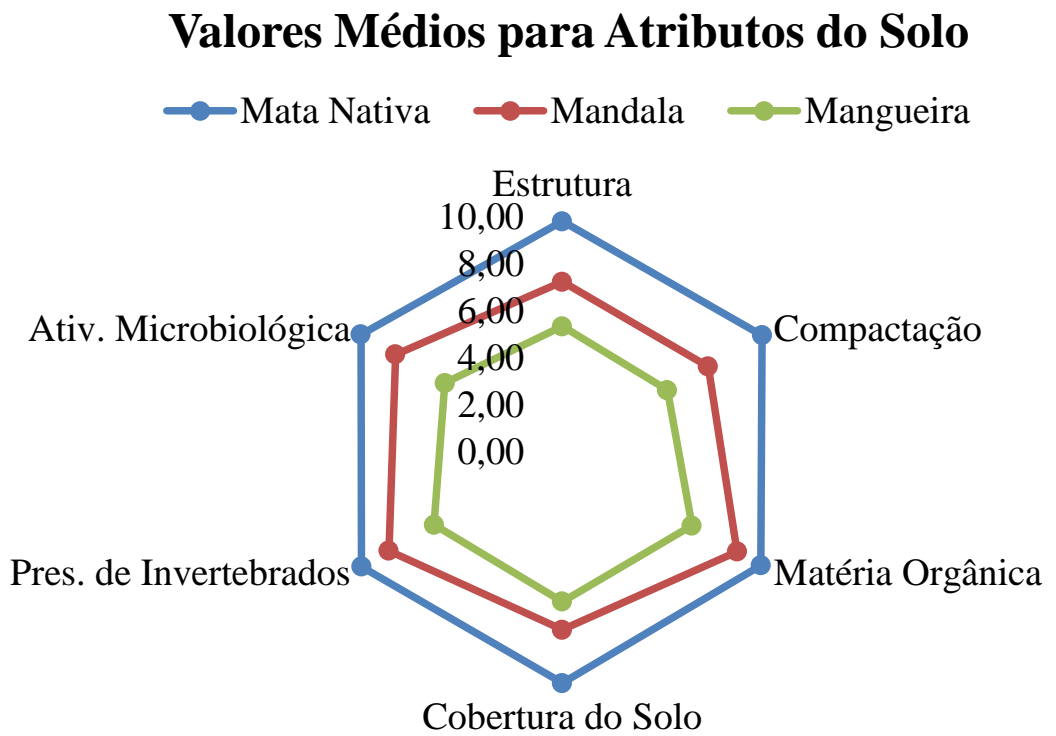

Figura 2. Valores referentes a qualidade dos solos dos subsistemas Mandala e Manga em referência a Mata nativa.

O subsistema manga obteve as médias mais baixas por se tratar de um sistema de cultivo que detém de menor interação, se comparado a mandala ou ecossistema natural. Em áreas que adotam técnicas conservacionistas, como a cobertura vegetal, apresentam solos bem estruturados, com pouca compactação, pois tais indicadores estão diretamente relacionados. A cobertura dos solos, como afirma Primavesi (2016), é fundamental na intenção de controlar a erosão acelerada, protegendo assim, o sistema macroporoso do solo. Nesses mesmos indicadores, Estrutura, Compactação e Cobertura do solo, o None obteve valores 5, 6 e 5 respectivamente. Os valores foram menores por diversos motivos, como o monocultivo, a única espécie presente na área, além do Manga, é o capim braquiária (Brachiaria decumbens), não há o incremento de cobertura vegetal, e como já citado, as poucas técnicas conservacionistas utilizadas na área foram adotadas a pouco tempo. Para os indicadores de Matéria orgânica, Presença de invertebrados e Atividade microbiológica, a Mandala ficou com média oito (8), já o Manga apresentou médias de 6, 6 e 5 respectivamente. Esses valores são também diretamente ligados às práticas utilizadas nas áreas. Os canteiros da mandala apresentaram cor marrom escuro por efeito da constante aplicação de matéria orgânica no solo, a comunidade microbiana do solo é pouco perturbada, assim apresenta maiores níveis de diversidade e estabilidade e contribui para maior vitalidade do agroecossistema (FERREIRA; WENDLAND; DIDONET, 2011). 
Esses resultados refletem como as práticas escolhidas e o histórico de uso influenciam diretamente na saúde e qualidade dos solos, Altieri e Nicholls (2002) apontam que os agroecossistemas diversificados seguem os princípios da sustentabilidade, a permanência e manutenção da capacidade produtiva do agroecossistemas, biodiversidade funcional flora e fauna.

Da mesma forma que o subsistema Mandala passa por um período de recuperação, porém já avançada, apresentando bons índices de qualidade dos solos, o subsistema None necessita de um manejo mais adequado com a situação que se encontra. Marzall e Almeida (1999), "relatam que indicador é um instrumento que permite mensurar as modificações nas características de um sistema, é uma ferramenta que permite a obtenção de informações sobre uma realidade", ou seja compreendendo em que ponto estamos, fica mais fácil decidir onde queremos ir.

\section{Análise convencional química e fertilidade}

$\mathrm{Na}$ tabela 2, observamos os valores referentes a caracterização química dos solos dos subsistemas Mandala e Mangueiras, e do Ecossistema natural de Mata Atlântica, localizados no CCHSA/UFPB.

Tabela 2. Caracterização química da fertilidade dos solos dos dois subsistemas Mandala, Manga e Mata.

\begin{tabular}{|c|c|c|c|c|c|c|c|c|c|c|c|c|c|c|c|c|}
\hline \multicolumn{15}{|c|}{ 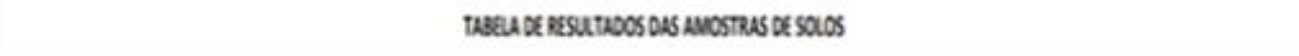 } & \multicolumn{2}{|c|}{ arteosowos } \\
\hline Avostra & 애 & $p$ & $x$ & $\mathbf{N}^{\circ}$ & $x+R^{2}$ & $N^{4}$ & $a^{4}$ & $4^{2}$ & $\$ 8$ & $\pi$ & $v$ & $n$ & mo. & Dentracio & $x$ & $\cos$ \\
\hline M/2018 & S.:0(12.25) & \multicolumn{2}{|c|}{$n / 4 n^{2}$} & \multicolumn{7}{|c|}{ conolyan' } & \multicolumn{2}{|c|}{$x$} & 24 & None elou Nines & anowlans 3 & $2 \sqrt{4}$ \\
\hline 237 & 5,64 & 22,51 & 40,95 & 0,05 & 5,61 & 0,05 & 5,10 & 280 & 8,05 & 13,66 & 58,95 & 0,62 & 64,41 & MATA & 0,105 & 37,36 \\
\hline 238 & 5,07 & 176,32 & 20,28 & 0,05 & 1,49 & 0,05 & 2,40 & 120 & 3,70 & 5,19 & 71,37 & 1,33 & 28,89 & NANGA & 0.052 & 16,75 \\
\hline 200 & 6,09 & 81,37 & 4,85 & 0,09 & 3,30 & 0,00 & 2,30 & 1,70 & 4,20 & 7,50 & 56,03 & 0,00 & 31,03 & MAVOALA & 0,115 & 18,00 \\
\hline
\end{tabular}

Observando os valores de $\mathrm{pH}$, Mata 5,64 teve acidez média de acordo com a classificação. Nessa condição pode possuir elevados teores de Al3+ (tóxico), boa disponibilidade de micronutrientes, porém, pode ocasionar baixa atividade de microorganismos (PREZOTTI et al., 2013). O pH da Manga 5,07 e da Mandalla 6,09 o que representa índices de acidez fraca. Provavelmente possui baixa presença de $\mathrm{Al} 3+$, boa disponibilidade de boro (B), disponibilidade intermediária dos demais micronutrientes, $\mathrm{pH}$ ideal para a maioria das culturas (PREZOTTI et al., 2013). 
O teor de fósforo $(\mathrm{P})$ para as plantas é uma medida relativa da quantidade do elemento no solo. O teor de $\mathrm{P}$ determinado na análise de solo é um valor relativo, que indica o teor de $\mathrm{P}$ "disponível" para as culturas. (PREZOTTI et al., 2013). resultados com valores mais altos para os subsistemas Mandala 81,37 e Manga 176,32, e valores baixos para Mata 22,51. Apesar da diferença entre os valores, de acordo com a classificação todos os valores de P são positivos para os subsistemas. Segundo Primavesi \& Primavesi (2018) o fósforo é o mineral mais necessário para o crescimento e a reprodução vegetal. A deficiência deste elemento é um tanto difícil de reconhecer, pois não existem sintomas muito expressivos.

Em relação aos valores de potássio $(\mathrm{K})$ o maior valor foi no subsistema Mandala 44,85, seguindo da mata com 40,95. De acordo com Prezotti et al., (2007), para culturas permanentes (Mata,Manga), anuais e hortaliças (Mandala), todos os valores estão abaixo da média. Primavesi \& primavesi (2018) salientam que, a deficiência em potássio torna a planta menos resistente às doenças bacterianas, menos resilientes e todo o desenvolvimento da planta é retardado.

Para cálcio (Ca) o maior valor da tabela foi da Mata nativa com 5,10, possuindo classificação alta, sendo um aspecto positivo. Os demais subsistemas estão com valores médios. Primavesi \& Primavesi (2018) citam que o cálcio é um elemento fundamental no metabolismo vegetal, ele no solo, está facilmente sujeito a lixiviação.

No que diz respeito ao magnésio $(\mathrm{Mg})$, também foi a Mata que obteve o maior valor 2,80, contudo, todos os subsistemas alcançaram classificação alta em relação desse elemento. Em relação ao magnésio, Primavesi \& Primavesi (2018) cita que é o principal fator na formação da clorofila e toma parte da na formação de todos os óleos e gorduras vegetais, formando ceras que protegem os vegetais contra a seca e condições desfavoráveis do solo. Por essas e outras razões, Prezotti et al., (2013) aponta que é importante que os teores de Ca e Mg estejam acima dos níveis adequados no solo, para o desenvolvimento pleno das culturas.

A Soma de Bases (SB), representa a soma das bases presentes no solo, ou seja, dos elementos $\mathrm{K}, \mathrm{Na} \mathrm{Ca}$ e $\mathrm{Mg}$. $\mathrm{Na}$ análise das amostras, a Mata foi quem sobressaiu com maior valor 8,05 e classificação alta. Os demais subsistemas estão entre valores médios.

Em relação a capacidade de troca de cátions pH 7,0 (CTC), é uma das variáveis mais importantes para a interpretação do potencial produtivo do solo. Indicando a quantidade total de cargas negativas que o solo poderia apresentar, se o seu $\mathrm{pH}$ fosse 7 (PREZOTTI et al., 2013). Na análise das amostras dos subsistemas, a Mata novamente foi quem alcançou o maior valor 13,66 e classificação alta. Os demais subsistemas estão entre os valores médios. A 
capacidade de troca iônica dos solos representa o aumento da capacidade de liberação de vários nutrientes, favorecendo a manutenção da fertilidade por um período prolongado (RONQUIM, 2010).

Na Saturação por bases (V), a análise das amostras demonstrou que os subsistemas estão entre os valores médios. A saturação por bases é um excelente indicativo das condições gerais de fertilidade do solo, sendo utilizada até como complemento na nomenclatura dos solos (RONQUIM, 2010).

Outra análise importante que foi realizada, foi a matéria orgânica (M.O). O teor de MO do solo é calculado multiplicando-se o teor de carbono do solo (carbono orgânico) pelo fator 1,72 (obtido pela divisão 100/58)(PREZOTTI et al., 2013). A amostra que obteve o maior valor foi a Mata 64,41 , porém os outros subsistemas tiveram também média alta. Machado e Machado Filho (2017) cita que a matéria orgânica desempenha múltiplas e positivas funções no solo, dentre elas: absorção e retenção de água; resiliência; reservatório de Carbono; berço de macro e microelementos. Com a mineralização da MO no solo, há liberação de bases que se encontravam imobilizadas nas cadeias carbônicas dos tecidos vegetais, que promovem a regulação do $\mathrm{pH}$, a disponibilidade de nutrientes, poder tampão e redução da toxidez (PREZOTTI et al., 2013).

\section{Conclusões}

O subsistema Mandala apresentou melhores níveis de sustentabilidade em referência Mata Nativa, já o subsistema Manga apresentou índices mais baixos, apontando que esse subsistema deve re-desenhar e manejar sua unidade, no enfoque dos princípios e técnicas agroecológicas, incrementando urgentemente a biodiversidade funcional.

A metodologia do trabalho se mostrou eficaz para diagnosticar a condição qualitativa do solo, diagnosticando as limitações e potencialidades de cada subsistema, apontando para as intervenções necessárias em cada um deles. Através da metodologia com indicadores é possível realizar o acompanhamento e o monitoramento das áreas, facilitando a tomada de decisão.

\section{Referências}

AITA, C. \& GIACOMINI, S. J. Decomposição e mineralização de nitrogênio de resíduos culturais de plantas de cobertura do solo solteiras e consorciadas. Revista Brasileira de Ciência do Solo, v.28, p.739-749, 2003. 
CARNEIRO, W. J. O.; SILVA, C. A.; MUNIZ, J. A. et al. Mineralização de nitrogênio em Latossolos adubados com resíduos orgânicos. Revista Brasileira de Ciência do Solo, v.37, p.715-725, 2013.

El Niño and La Niña. Disponível em: < http://www.stormfax.com/elnino.htm $>$ Acesso em 3 nov. 2018.

EMPRESA DE PESQUISA AGROPECUÁRIA - EMBRAPA. Centro Nacional de Pesquisas de Solos. Sistema brasileiro de classificação de solos. 2.ed. Rio de Janeiro, 2006. 306p.

FERREIRA, D. F. Sisvar: a guide for its bootstrap procedures in multiple comparisons. Ciência e Agrotecnologia, Lavras, v.38, p.109-112, 2014.

JACKSON, M. L. Chemical composition of soil. In: BEAR, F. E., ed. Chemistry of the soil. 2.ed. New York: Reinhold, 1964. p.71-141.

MALAVOLTA, E. Manual de nutrição mineral de plantas. São Paulo: Agronômica Ceres, 2006. $638 \mathrm{p}$.

POTTKER, D. \& TEDESCO, M. J. Efeito do tipo e tempo de incubação sobre a mineralização da matéria orgânica e nitrogênio total em solos do Rio Grande do Sul. Revista Brasileira de Ciência do Solo, v.3, p.20-24, 1979.

RAIJ, B. V. Gesso na agricultura. Campinas, SP: IAC. 2008. 233 p.

RIBEIRO, A. C.; GUIMARÃES, P. T. G.; ALVAREZ, V. V. H. Recomendação para o uso de corretivos e fertilizantes em Minas Gerais: $5^{\text {a }}$ aproximação. Viçosa, MG: Comissão de Fertilidade do Solo do Estado de Minas Gerais, 1999. 359 p.

SILVA, F. C.; ABREU, M. F.; PÉREZ, D. V. et al. Métodos de análises químicas para avaliação de fertilidade do solo. In: SILVA, F. C. (Ed. técnico). Manual de análises químicas de solos, plantas e fertilizantes. Brasília, Embrapa comunicação para transferência de tecnologia, 2009. p.107-184.

VETTORI, L. Ferro “livre” por cálculo. In: CONGRESSO BRASILEIRO DE CIÊNCIA DO SOLO, 15., Campinas, 1975. Anais. Campinas: Sociedade Brasileira de Ciência do Solo, 1976. p.127-128. 